\title{
Etude dynamique d'un train de pêche complet
}

\author{
Dominique Marichal* — Benoît Vincent*** \\ * Ecole Centrale Nantes \\ 1 rue de la Noë, F-44300 Nantes \\ dominique.marichal@ec-nantes..fr \\ ** IFREMER \\ 8 rue François Toullec, F-56100 Lorient \\ benoit.vincent@ifremer.fr
}

\begin{abstract}
RÉSUMÉ. Nous présentons l'étude dynamique d'un train de pêche complet. Le chalutier est modélisé par les équations classiques des théories de tenue à la mer et de manœuvrabilité des navires. Il est soumis à l'action des funes. Les funes et les filets constituent un problème de couplage fluide-structure complexe. Le filet est une structure souple dont la forme dépend des efforts auxquels il est soumis. Mais ces efforts dépendent eux-même de la forme prise par les mailles. Les panneaux sont des profils portants très particuliers. Nous avons étudié en détail un type de panneau. Lorsque tous ces éléments sont rassemblés, "il suffit " d'écrire les équations de la dynamique pour chacun des objets considérés. Une résolution à pas de temps permet de suivre la déformation du train de pêche complet, l'évolution des tensions en tout point du chalut et de son gréement, les mouvements du chalutier...
\end{abstract}

ABSTRACT. We present a complete numerical model of a full trawl gear and the vessel. The trawler is modelled by equations and hydrodynamic coefficients which are classically used for ship behaviour and ship manoeuvrability studies. It is submitted to the action of the warps. The warps and the net are part of a complex fluid-structure coupling problem. The net is a supple structure and its shape depends on the forces that are acting on it. Now, these forces themselves depend of the net meshes shape. The otter boards are most particular wings. We have studied one particular type. Then all these elements have been put together by writing their dynamics equations. A time step method enables the calculation of the shape, the forces and the motion of each element in the whole system.

MOTS-CLÉS : train de pêche, chalut, filet, structure réticulée souple immergée, panneau, manoeuvrabilité, accident de pêche, croche.

KEYWORDS : trawl gear, net, immersed supple structure, otter board, manoeuvrabilty, fishing accident, obstruction.

Revue. Volume $\mathrm{X}-\mathrm{n}^{\circ} \mathrm{x} /$ année, pages 1 à $\mathrm{X}$ 


\section{Introduction}

\subsection{Etat de l'art}

Les “Arts traînants” et le chalutage sont des techniques de pêche ancestrales utilisant comme constituant principal un filet. Pendant longtemps, la validation des idées nouvelles résultait seulement d'une augmentation de la capture, ou d'une amélioration des conditions de travail. Ce qui se " passait » sous l'eau était pratiquement inconnu. La construction de moyens d'essais importants a permis l'observation de modèles réduits de train de pêche. Malgré des difficultés liées aux effets d'échelle, ces expériences ont apporté de nombreux renseignements sur la forme prise par les chaluts suivant les conditions de mise en œuvre. Le développement d'équipements de mesure sous-marins performants a permis de compléter ces informations par des observations et des résultats quantitatifs de plus en plus précis dans les conditions d'utilisation réelle.

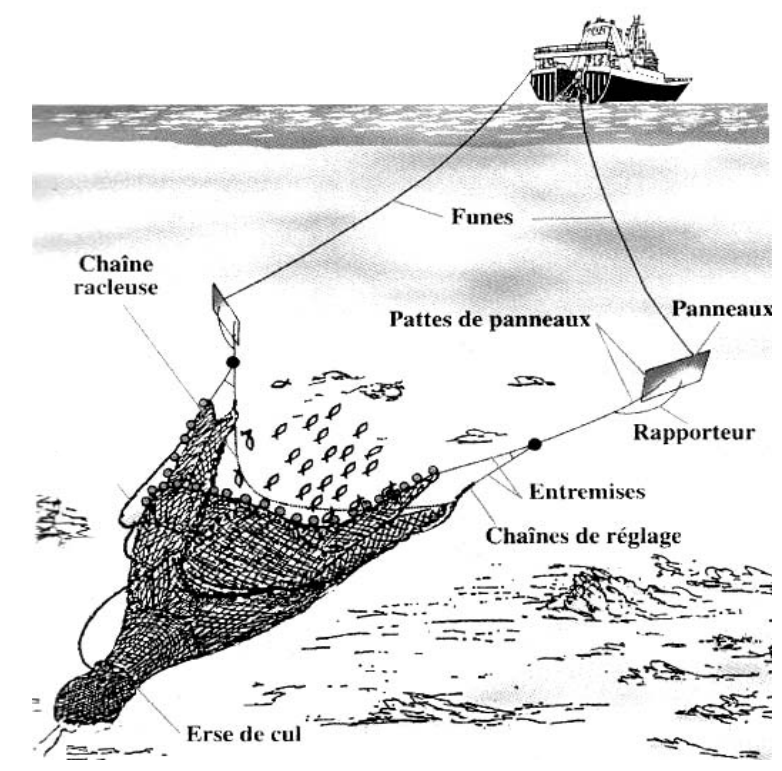

Figure 1. Principaux éléments d'un train de pêche (George, 2005)

Cependant la complexité des études expérimentales limite leur utilisation à quelques cas spécifiques. C'est pourquoi nous nous attachons depuis plusieurs années à développer l'étude numérique des trains de pêche. Les objectifs de ces études sont multiples. Une meilleure connaissance du comportement du train de pêche permet sûrement d'en améliorer l'efficacité, non pas tant en augmentation des captures qu'en diminution des coûts de fabrication, d'exploitation et d'entretien. Mais c'est surtout dans un contexte de respect de l'environnement et des ressources 
marines que ces outils numériques facilitent les recherches visant à améliorer la sélectivité des chaluts ou à diminuer l'impact des trains de pêche sur le fond marin. Enfin, la simulation de manœuvres ou d'accidents fréquemment rencontrés au cours de campagnes de pêche pourra également améliorer la sécurité des pêcheurs.

\section{Méthode générale pour l'étude dynamique d'un filet immergé}

\subsection{Un problème de couplage fluide-structure original et complexe}

Un chalut est une surface souple réticulée et immergée, dont l'interaction avec l'écoulement est particulièrement complexe :

- le comportement hydrodynamique des mailles de filet est très mal connu. Au passage d'une maille, le fluide est-il accéléré ou bien au contraire ralenti ou encore dévié ? (problème dit de " filtration » des mailles). Quelles sont les influences des angles d'ouverture et d'incidence d'une maille sur les efforts hydrodynamiques auxquels elle est soumise ? Quelle interaction existe-t-il entre une maille et ses voisines?

- un filet est une structure infiniment souple, qui n'a pas de forme propre, seule l'action de forces extérieures lui permet de prendre une certaine configuration. Mais, dans le cas de filets immergés, ces forces dépendent elles-mêmes de la forme prise par le filet, nous devons donc traiter un véritable problème de couplage fluidestructure.

- une surface réticulée est par définition constituée par un matériau discontinu et anisotrope (la façon d'assembler les mailles crée des directions privilégiées pour la transmission des efforts et des déformations). De plus, certaines mailles très tendues peuvent se fermer complètement et devenir ainsi des fils pratiquement inextensibles, d'autres peuvent être complètement détendues et "flotter" sans forme vraiment bien définie.

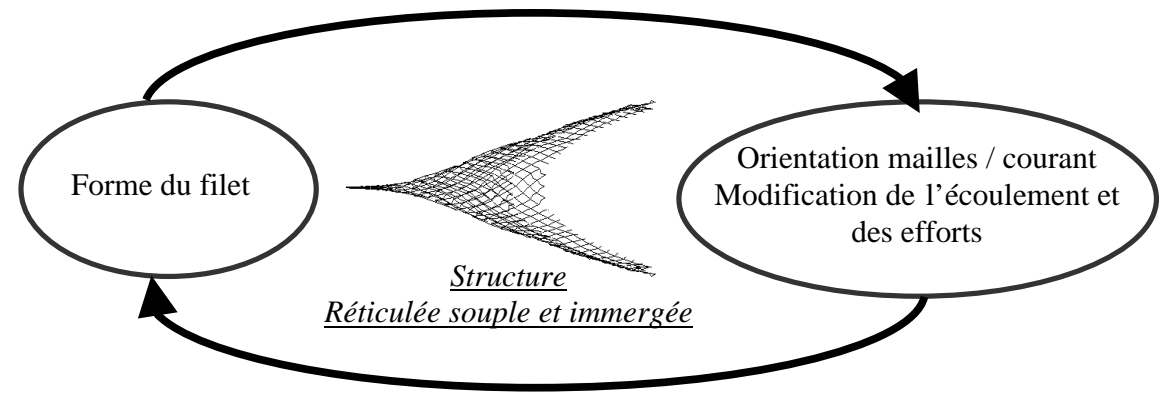

Figure 2. Un problème complexe de couplage fluide-structure 
Des solutions très partielles et approchées de ce problème complexe ont pu être données, à l'aide de séries systématiques d'essais et d'extrapolations, V.A. Belov par exemple (Belov, 1987). Cette démarche ne permet pas l'innovation car elle n'est applicable qu'à des filets de même type que ceux déjà essayés. La méthode que nous proposons nous semble une approche originale et plus générale. Elle est facilement adaptable à de nombreux problèmes.

\subsection{Méthode générale pour l'étude dynamique d'un filet immergé}

Un chalut réel comporte un nombre de mailles extrêmement important. Nous avons donc introduit une méthode de globalisation : on remplace un groupe de mailles réelles par une maille virtuelle. Son coté est défini par le nombre de mailles globalisées. Le diamètre de ses cotés est calculé de façon à ce qu'elle génère les mêmes efforts hydrodynamiques que les mailles remplacées. Puis, la maille globalisée est représentée par un ensemble de barres cylindriques rigides reliées entre elles par des liaisons rotules. Des liaisons rotules intermédiaires rendent compte de la souplesse des cotés de maille.
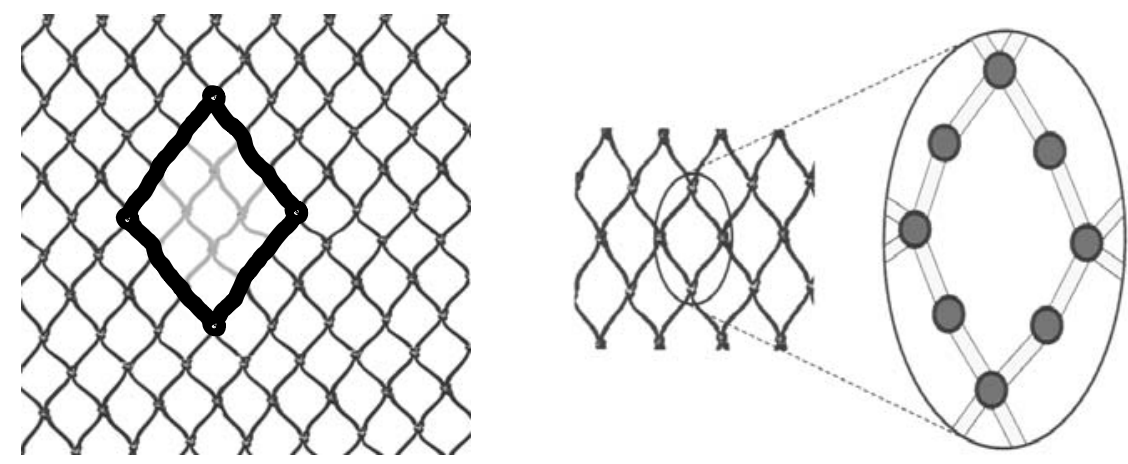

Figure 3. Globalisation du maillage et modélisation de la maille globalisée

Les efforts hydrodynamiques agissant sur chacun des cotés des mailles sont calculés comme si ceux-ci étaient des cylindres circulaires isolés. Ils sont décomposés en une traînée normale au coté de maille et un effort de frottement tangentiel. Les valeurs des coefficients de traînée et de frottement ont été obtenues par des expériences en canal à circulation d'eau et à l'aide d'une modélisation CFD de l'écoulement à travers une alèse plane : $C_{d}=1,2$ et $f=0,1$ (Marichal, 2005).

$$
\mathbf{T}_{\mathbf{p}}=-\frac{1}{2} \rho d \operatorname{lC}_{d} \mathbf{V}_{\mathbf{p}}\left\|\mathbf{V}_{\mathbf{p}}\right\| \quad \mathbf{F}_{\mathbf{t}}=-\frac{1}{2} \rho d l f C_{d} \mathbf{V}_{\mathbf{t}}\left\|\mathbf{V}_{\mathbf{t}}\right\|
$$


Chaque fil exerce une tension $\mathrm{t}_{\mathrm{ij}}$ sur les nœuds i et $\mathrm{j}$ situés à ses deux extrémités. Ces tensions sont liées aux cordonnées des nœuds par l'élasticité des cotés de mailles.

$$
l_{i j}=l_{0_{i j}}\left(1+\chi \mathbf{t}_{i j}\right) \quad l_{\mathrm{ij}} \text { longueur non tendue et } \chi \text { élasticité du coté de maille. }
$$

De plus, les cotés de mailles sont des fils infiniment souples et ne peuvent pas transmettre un effort de compression. Nous imposons donc que la tension d'un fil est nulle si la distance qui sépare ses deux extrémités est plus courte que sa longueur non tendue.

Certains nœuds sont soumis à des forces ponctuelles $\mathbf{L}_{i}$. Les nœuds liés aux flotteurs sont soumis à une force verticale (le poids apparent des flotteurs), et une force de traînée qu’on exprime en utilisant le coefficient de traînée d'une sphère $(0,45)$. De même, les lests peuvent être en contact avec le sol. Il apparaît une force de frottement. Le coefficient de frottement dépend beaucoup de la nature du sol.

Finalement, en notant $k$ le nombre de nœuds adjacents au nœud $i$ et $n_{1}(i), \ldots, n_{k}(i)$ leurs numéros, les équations de la dynamique de chacun des nœuds peuvent s’écrire sous la forme générale :

$$
\boldsymbol{\gamma}_{i}=\frac{1}{m_{i}} \sum_{j=n_{1}(j)}^{n_{k}(j)}\left\{\mathbf{t}_{i j}+\frac{1}{2}\left(\mathbf{T}_{p_{i j}}+\mathbf{F}_{t_{i j}}\right)+\mathbf{L}_{i}\right\}
$$

Nous obtenons ainsi un système d'équations différentielles du second ordre qui peut être facilement intégré en utilisant une méthode de Runge-Kutta.

\section{Modélisation des efforts hydrodynamiques agissant sur un panneau}

Les panneaux de pêche sont des surfaces portantes destinées à créer une force orthogonale à la vitesse d'avance afin d'assurer l'ouverture horizontale du filet. Pour des raisons de solidité et de mise en œuvre, et bien que les plus élaborés utilisent des principes issus de la théorie des ailes, leur forme est assez différente de celle d'une aile classique.

La connaissance des efforts hydrodynamiques auxquels ils sont soumis à longtemps été très empirique. Les bassins d'essais de chalut ont permis de mesurer, avec une précision limitée, les efforts principaux (portance et traînée). Les méthodes CFD permettent maintenant de déterminer le torseur complet des efforts hydrodynamiques stationnaires et instationnaires agissant sur un panneau de chalut (Marichal, 2006). Il s’agit d'une étude complexe que nous résumons rapidement ici pour le cas particulier les panneaux du NO Gwendrez (chalutier de recherche IFREMER). 


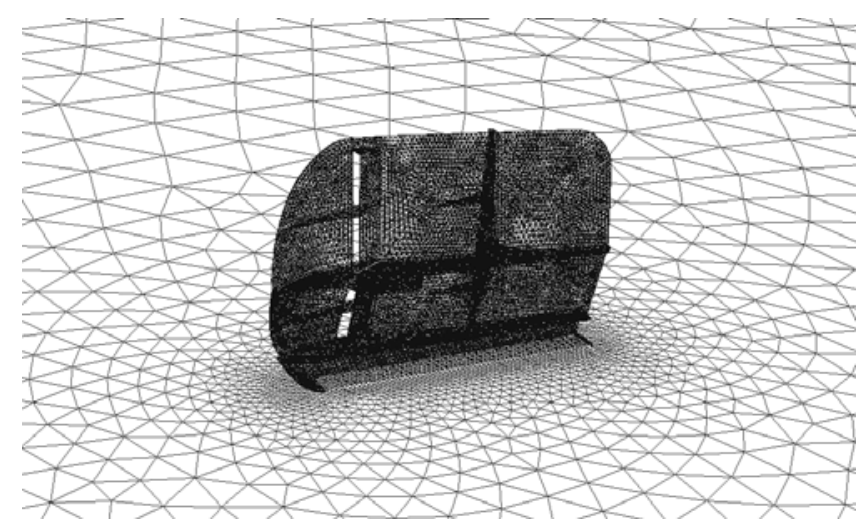

Figure 4. Maillage du panneau et du fond (roulis $0^{\circ}$ )

Pour le calcul des efforts hydrodynamiques, on considère que les paramètres de positions (lacet, tangage, roulis), les vitesses et les accélérations sont des paramètres indépendants. Bien que ces paramètres soient liés entre eux par des relations de dérivation - plus ou moins simples - par rapport au temps, un panneau réel peut avoir à un instant $t$ la même position avec des vitesses et accélérations totalement différentes. Nous pouvons donc écrire que chacune des composantes du torseur des efforts hydrodynamique est une fonction des 18 paramètres de position, vitesse et accélération. On linéarise l'expression de ces efforts en développant ces fonctions inconnues en séries de Taylor, autour de la position nominale du panneau et limitées au premier ordre. Ainsi une composante du torseur des efforts peut s'écrire :

$$
X=X_{0}+X_{u} u+X_{v} v+X_{w} w+X_{\dot{u}} \dot{u}+X_{\dot{v}} \dot{v}+X_{\dot{v}} \dot{w}+X_{\ddot{u}} \ddot{u}+X_{\ddot{v}} \ddot{v}+X_{\ddot{w}} \ddot{w}
$$

Dans un premier temps nous calculons les composantes stationnaires pour différentes combinaisons d'angles de lacet, tangage et roulis pour une vitesse de l'écoulement relatif de $1 \mathrm{~m} / \mathrm{s}$ (mouvement relatif permanent).

Les figures 5 présentent les variations de ces coefficients en fonction des angles de lacet et roulis pour un angle de tangage nul. Nous avons obtenu le même type de variations pour des valeurs de tangage de plus et moins $15^{\circ}$.

Puis nous imposons au panneau, placé dans l'écoulement relatif défini par ses orientations, une accélération constante successivement pour chacun des paramètres $\left(1 \mathrm{~m} / \mathrm{s}^{2}\right.$ ou $\left.1 \mathrm{rd} / \mathrm{s}^{2}\right)$. Nous lissons par une droite la courbe d'évolution de la composante considérée au voisinage de zéro. Le décalage entre la valeur à l'origine de cette droite et la valeur stationnaire correspond aux effets de l'inertie ajoutée. (Figure 6) 

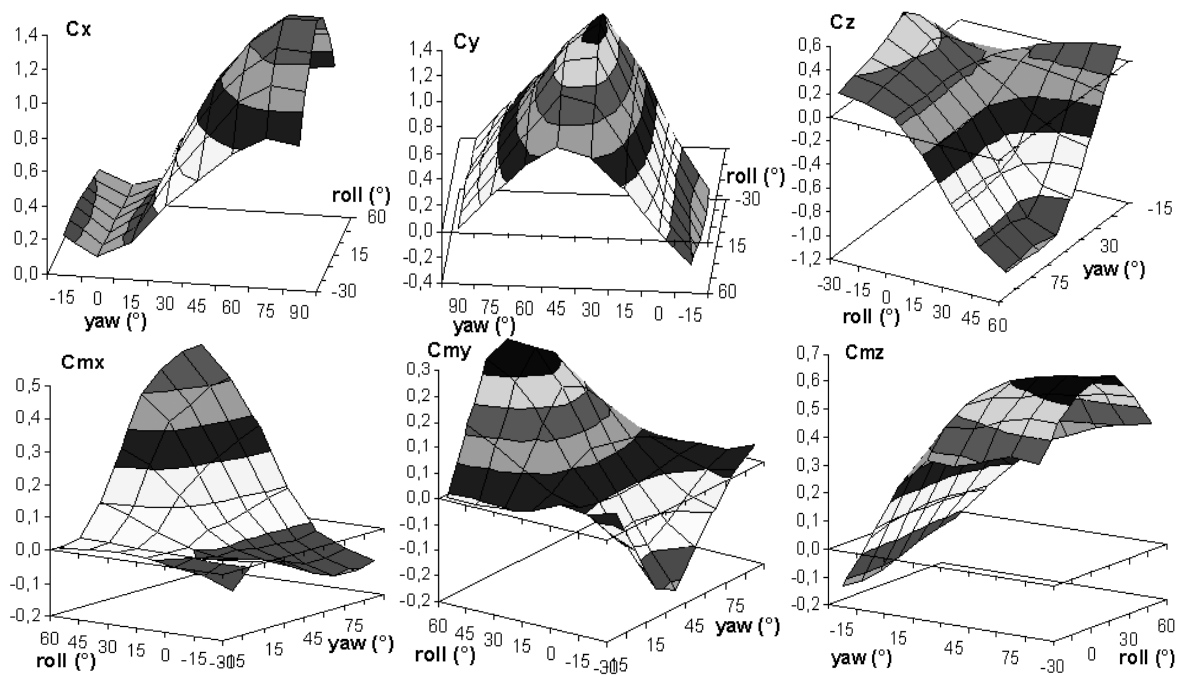

Figure 5. Variation des coefficients hydrodynamiques stationnaires (tangage $0^{\circ}$ )

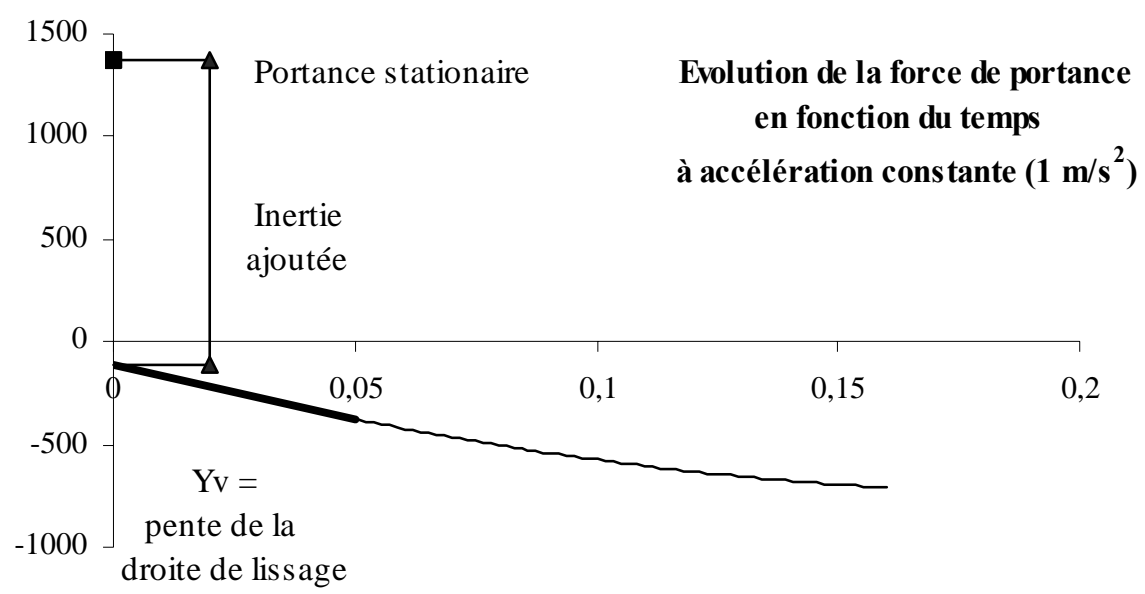

Figure 6. Détermination des efforts instationnaires (roul. $0^{\circ}$ - lacet $30^{\circ}$ - tang. $0^{\circ}$ )

Enfin le tableau 1 regroupe les 42 coefficients hydrodynamiques du panneau pour une des 170 positions étudiées : lacet $30^{\circ}$, tangage $0^{\circ}$ et roulis $0^{\circ}$. 
$\mathrm{X}_{\mathrm{u}}=-297 \quad \mathrm{X}_{\mathrm{v}}=-678 \mathrm{X}_{\mathrm{w}}=-73$

$\mathrm{Y}_{\mathrm{u}}=893 \mathrm{Y}_{\mathrm{v}}=-5659 \mathrm{Y}_{\mathrm{w}}=-505$

$\mathrm{Z}_{\mathrm{u}}=12 \quad \mathrm{Z}_{\mathrm{v}}=-237 \mathrm{Z}_{\mathrm{w}}=-786$

$\mathrm{K}_{\mathrm{u}}=-13 \quad \mathrm{~K}_{\mathrm{v}}=123 \quad \mathrm{~K}_{\mathrm{w}}=-318$

$\mathrm{M}_{\mathrm{u}}=-13 \quad \mathrm{M}_{\mathrm{v}}=-176 \quad \mathrm{M}_{\mathrm{w}}=-507$

$\mathrm{N}_{\mathrm{u}}=-768 \mathrm{~N}_{\mathrm{v}}=4362 \mathrm{~N}_{\mathrm{w}}=401$

\begin{tabular}{|l|l|l|}
\hline $\mathrm{X}_{\mathrm{up}}=-53$ & $\mathrm{X}_{\mathrm{vp}}=43$ & $\mathrm{X}_{\mathrm{wp}}=-3$ \\
$\mathrm{Y}_{\mathrm{up}}=34$ & $\mathrm{Y}_{\mathrm{vp}}=-1476$ & $\mathrm{Y}_{\mathrm{wp}}=-69$ \\
$\mathrm{Z}_{\mathrm{up}}=-3$ & $\mathrm{Z}_{\mathrm{vp}}=-70$ & $\mathrm{Z}_{\mathrm{wp}}=-173$ \\
$\mathrm{~K}_{\mathrm{up}}=0$ & $\mathrm{~K}_{\mathrm{vp}}=115$ & $\mathrm{~K}_{\mathrm{wp}}=-40$ \\
$\mathrm{M}_{\mathrm{up}}=0$ & $\mathrm{M}_{\mathrm{vp}}=-55$ & $\mathrm{M}_{\mathrm{wp}}=-153$ \\
$\mathrm{~N}_{\mathrm{up}}=-1124$ & $\mathrm{~N}_{\mathrm{vp}}=479$ & $\mathrm{~N}_{\mathrm{wp}}=-972$ \\
\hline
\end{tabular}

\begin{tabular}{|l|ll|l}
\hline $\mathrm{X}_{\mathrm{p}}=44$ & $\mathrm{X}_{\mathrm{q}}=$ & -14 & $\mathrm{X}_{\mathrm{r}}=50$
\end{tabular}

$\mathrm{Y}_{\mathrm{p}}=-214 \mathrm{Y}_{\mathrm{q}}=-499 \mathrm{Y}_{\mathrm{r}}=7067$

$\mathrm{Z}_{\mathrm{p}}=-271 \mathrm{Z}_{\mathrm{q}}=-769 \mathrm{Z}_{\mathrm{r}}=226$

$\mathrm{K}_{\mathrm{p}}=-476 \mathrm{~K}_{\mathrm{q}}=-349 \mathrm{~K}_{\mathrm{r}}=-206$

$\mathrm{M}_{\mathrm{p}}=-167 \mathrm{M}_{\mathrm{q}}=-728 \mathrm{M}_{\mathrm{r}}=142$

$\mathrm{N}_{\mathrm{p}}=281 \quad \mathrm{~N}_{\mathrm{q}}=462 \quad \mathrm{~N}_{\mathrm{r}}=-6751$

\begin{tabular}{|l|l|l|}
\hline $\mathrm{X}_{\mathrm{pp}}=0$ & $\mathrm{X}_{\mathrm{qp}}=-1$ & $\mathrm{X}_{\mathrm{rp}}=-97$ \\
$\mathrm{Y}_{\mathrm{pp}}=118$ & $\mathrm{Y}_{\mathrm{qp}}=-54$ & $\mathrm{Y}_{\mathrm{rp}}=1483$ \\
$\mathrm{Z}_{\mathrm{pp}}=-41$ & $\mathrm{Z}_{\mathrm{qp}}=-151$ & $\mathrm{Z}_{\mathrm{rp}}=58$ \\
$\mathrm{~K}_{\mathrm{pp}}=-104$ & $\mathrm{~K}_{\mathrm{qp}}=-43$ & $\mathrm{~K}_{\mathrm{rp}}=-118$ \\
$\mathrm{M}_{\mathrm{pp}}=-45$ & $\mathrm{M}_{\mathrm{qp}}=-169$ & $\mathrm{M}_{\mathrm{rp}}=49$ \\
$\mathrm{~N}_{\mathrm{pp}}=-1152$ & $\mathrm{~N}_{\mathrm{qp}}=-983$ & $\mathrm{~N}_{\mathrm{rp}}=-2719$ \\
\hline
\end{tabular}

Tableau 1. Coefficients hydrodynamiques du panneau (Roulis $0^{\circ}$ - Lacet $30^{\circ}$ )

\section{Etude des mouvements du chalutier}

Nous étudions les mouvements du chalutier en utilisant les équations proposées par M.A. Abkowitz (1969). Les équations présentées ci-dessous correspondent au cas d'un mouvement dans un plan horizontal (celles que nous pouvons utiliser pour l'étude de la manoeuvrabilité du train de pêche en eau calme). Mais nous pouvons aussi utiliser le système d'équations correspondant à un mouvement quelconque avec six degrés de liberté (cavalement, embardée, pilonnement, lacet, roulis et tangage). Ces équations sont linéarisées autour d’une position d'équilibre définie comme le navire avançant en ligne droite à vitesse constante.

$$
\begin{aligned}
& \left(X_{\dot{u}}-m\right) \dot{u}+X_{u}\left(u-U_{0}\right)+X_{\dot{v}} \dot{v}+X_{r} r+X_{\dot{r}} \dot{r}+X_{r} r=0 \\
& Y_{\dot{u}} \dot{u}+Y_{u}\left(u-U_{0}\right)+\left(Y_{\dot{v}}-m\right) \dot{v}+Y_{v} v+\left(Y_{\dot{r}}-m x_{G}\right) \dot{r}+\left(Y_{r}-m U_{0}\right) r=0 \\
& N_{\dot{u}} \dot{u}+N_{u}\left(u-U_{0}\right)+\left(N_{v}-m x_{G}\right) \dot{v}+N_{\dot{v}} \dot{v}+N_{v} v+\left(N_{r}-I_{Z}\right) \dot{r}+\left(N_{r}-m x_{G} U_{0}\right) r=0
\end{aligned}
$$

Le calcul des différents coefficients hydrodynamiques du navire a été fait pour le No Gwendrez (chalutier de recherche IFREMER) à l'aide d'un code « fluide parfait ». Ce code ne prend donc pas en compte les effets de la viscosité. Mais l'influence la plus importante de la viscosité se trouve dans la résistance à l'avancement. Celle-ci est compensée par la poussée de l’hélice. C'est pourquoi ni 
la traînée du bateau ni la poussée de l’hélice n’interviennent explicitement dans ces équations.

Si le chalutier est soumis à d'autres actions extérieures qui modifient son état d'équilibre dynamique, les équations précédentes sont utilisables à condition d'y introduire les forces correspondantes. La liaison entre le navire et le train de pêche est donc modélisée en exprimant que l'action des funes sur le chalutier est due à la tension de leur premier élément et que le mouvement de l'extrémité des funes est imposé par le mouvement du bateau.

Pour le navire seul et avançant à vitesse constante, nous avons déjà rappelé que la poussée de l’hélice compensait les efforts de traînée. Mais lorsque le chalutier est en pêche, l’hélice doit aussi compenser la traînée du chalut et de son gréement. Pour évaluer cet effort supplémentaire, il est nécessaire de faire un premier calcul en imposant au chalutier une vitesse constante égale à la vitesse nominale du chalutage. Lorsque l'équilibre du chalutier est perturbé, particulièrement en vitesse et en tangage, la poussée de l'hélice peut varier. Nous ne sommes pas encore en mesure de tenir compte de ces modifications de poussée. Nous maintenons donc celle-ci généralement constante. Nous pouvons aussi introduire une modulation de la poussée pour représenter, empiriquement et assez grossièrement, l'action du capitaine sur sa barre d'avance. Nous ne disposons pas d'études précises sur les efforts engendrés par le safran du No Gwendrez. Pour provoquer une giration du chalutier, on applique donc une force transversale au centre du safran. Cette force est déterminée empiriquement pour obtenir le rayon de giration souhaité.

\section{Exemples}

\subsection{Equilibre du chalut}

La figure 7 présente la forme de l'entrée d'un chalut remorqué à vitesse constante.

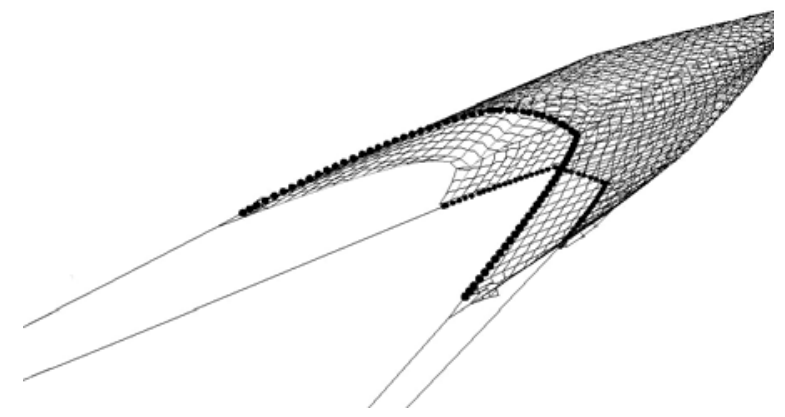

Figures 7. Chalut remorqué à vitesse constante 


\subsection{Simulation d'une croche}

La croche est un événement ponctuel particulièrement dangereux pour les arts traînants. Elle peut non seulement causer des avaries à l'engin mais aussi , dans des conditions extrêmes, compromettre la sécurité de l'équipage et du navire. Elle est due au blocage d'un élément du train de pêche par un obstacle. Nous présentons ici l'étude de croche comme étant l'arrêt brutal d'un point du bourrelet. Pour la simuler nous figeons les coordonnées du nœud correspondant. Dans cet exemple nous maintenons la poussée de l'hélice constante (le pilote ne s'est pas rendu compte de l'accident !). Il serait possible de prévoir une réaction du pilote qui arrêterait la poussée de l'hélice avec un certain retard. Les figures 8 et 9 présentent l'évolution de la forme du chalut durant la croche. L'action des flotteurs soulève la corde de dos.

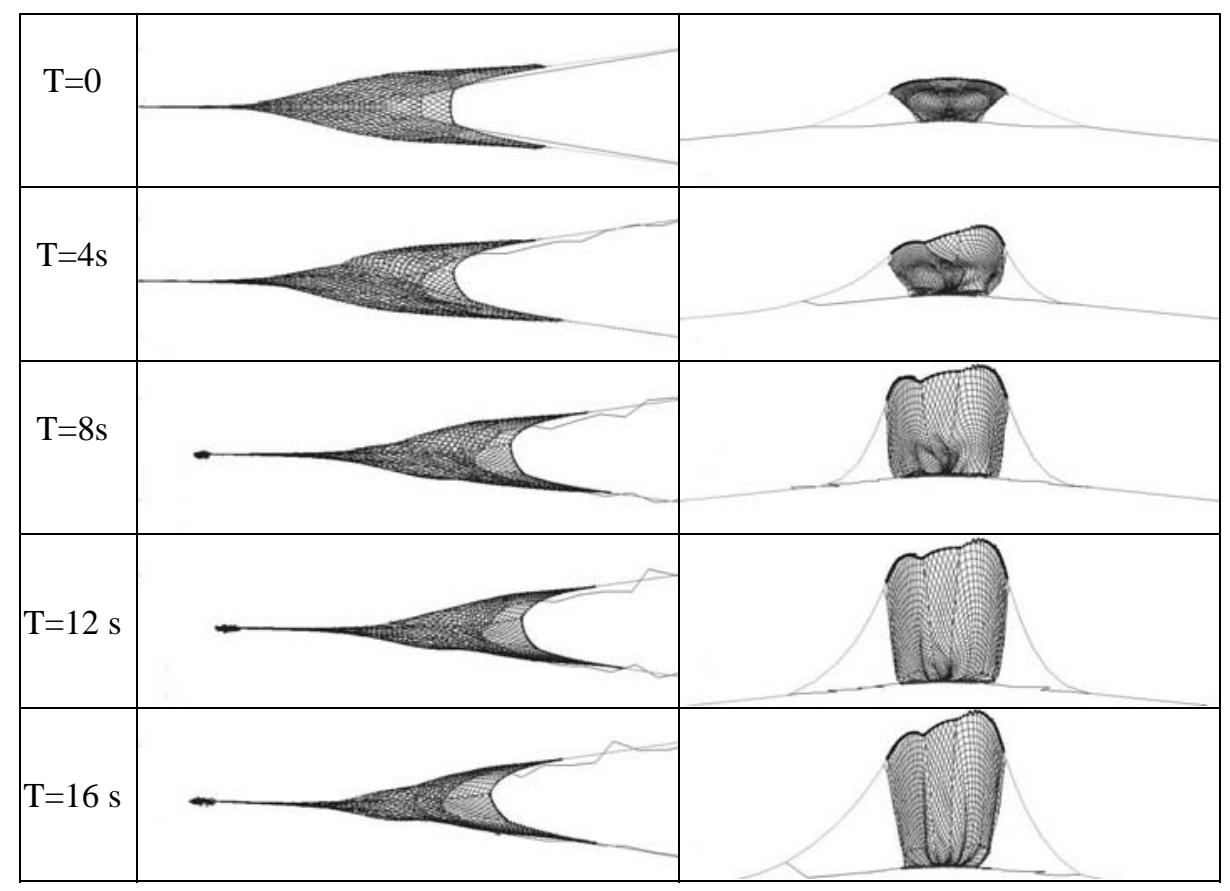

Figures 8. «Chronophotographie » d'une croche dissymétrique 

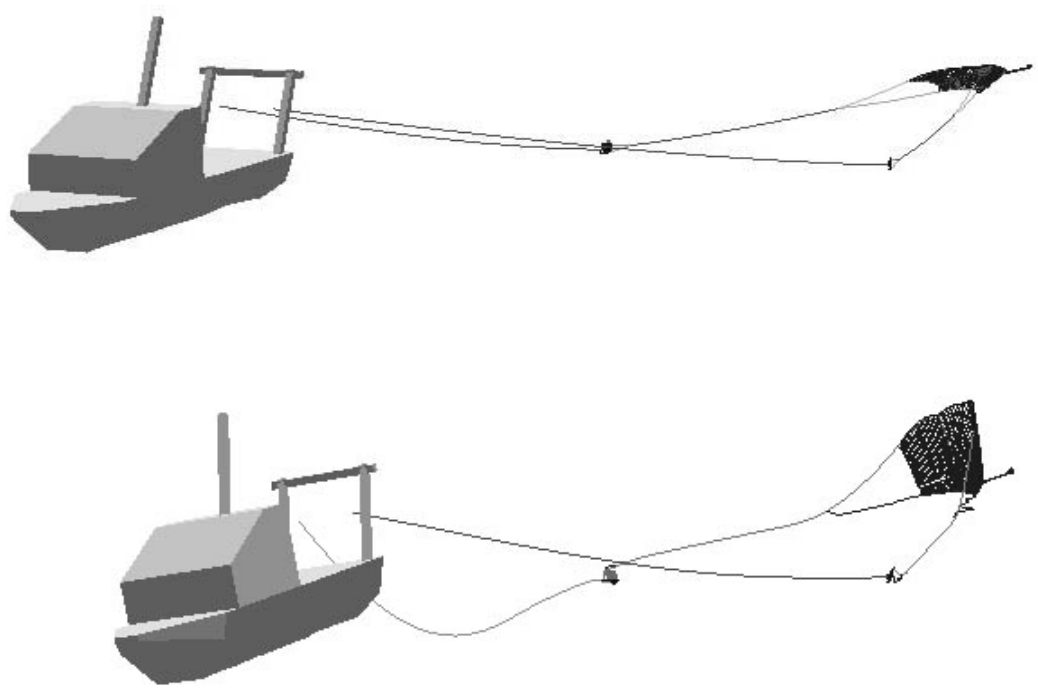

Figure 9. Aspect global du train de pêche avant la croche et à la fin de la croche

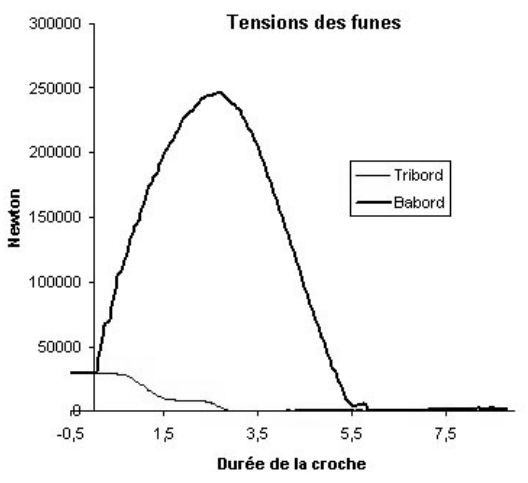

Figure 10. Tensions des funes

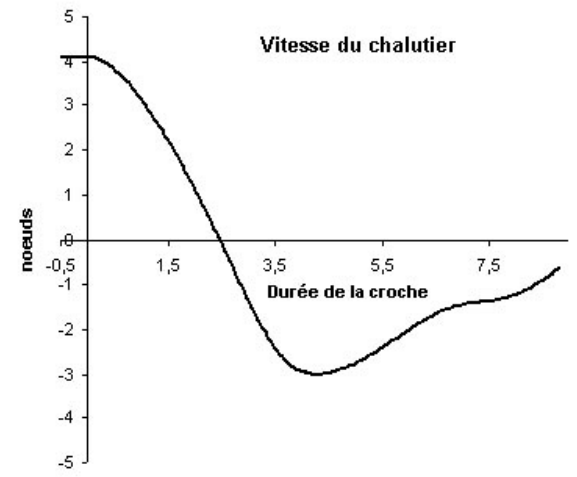

Figure 11.Vitesse du chalutier

La tension de la fune tribord croît très rapidement et de manière très importante (figure 10).Elle est multipliée par un facteur 8 en 2,5 secondes ! Par contre, la fune bâbord se détend complètement. Dans le même temps, l'importance de la tension la tension tribord arrête complètement le chalutier et le fait même reculer malgré la poussée de l’hélice (figure 11). 


\section{Conclusion}

L’exemple proposé montre l'efficacité de notre méthode. Les résultats obtenus sont satisfaisants et conformes aux observations pratiques. Mais il met aussi en évidence que, malgré l'importance du travail déjà réalisé, il reste encore beaucoup à faire. Il faut sans doute affiner le calcul des coefficients hydrodynamiques du chalutier, en particulier, les coefficients d'amortissement si on veut obtenir une simulation précise de la seconde phase de la croche. Les méthodes employées sont lourdes et il n'est pas encore possible d'envisager de les appliquer couramment. Enfin, nous espérons pouvoir valider certains résultats par des essais à la mer.

\section{Bibliographie}

Abkowitz M.A., Stability and Motion Control of Ocean Vehicles, Course of Instruction in Ocean Engineering, Massachusetts Institute of Technology, 1969

Belov V.A., Engins de pêche remorqués, Moscou, Editions Agropromizdat, 1987.

Bessonneau J.-S., Etude dynamique de surfaces réticulées souples et immergées, Thèse de Doctorat, Université de Nantes, Ecole Centrale de Nantes, 1997.

George J.P., Les croches et les arts traînants, Editions IFREMER, 2005.

Landweber L. and Protter M.H., «The shape of a light flexible cable in a uniform current », Journal of Applied Mechanics, June 1947, p. 121-126

Marichal D., «Can the CFD help in the conception of the fishing gears ? Study of hydrodynamic forces acting on nets », CANCAM 2005, Montréal, 30 mai - 2 juin 2005.

Marichal D. Etude des efforts hydrodynamiques instationnaires sur les panneaux de chalut Etude pour Ifremer Rapport contrat 20042 2003141, 2006

Théret F., Etude de l'équilibre de surfaces réticulées placées dans un courant uniforme, Thèse de Doctorat, Université de Nantes, Ecole Centrale de Nantes 